Note: This is a draft of a paper submitted for publication. Contents of this paper should not be quoted or referred to without permission of the author(s).

To be presented at Gettering and Defect Engineering in Semiconductor Technology Conference, Wulkow/Neuhardenberg, Germany September 2-7, 1995 and published in Proceedings

\title{
DETERMINATION OF INTERFACE STRUCTURE AND BONDING BY Z-CONTRAST STEM
}

S. J. Pennycook, N. D. Browning, M. M. McGibbon, A. J. McGibbon, M. F. Chisholm, and D. E. Jesson

Solid State Division

Oak Ridge National Laboratory, Oak Ridge, Tennessee 37831-6030

"The submitted manuscript has been authored

by a contractor of the U.S. Govemment under

contract No. DE-AC05-84OR21400.

Accordingly, the U.S. Government retains

nonexclusive, royalty-free license to publish or

reproduce the published form of this

contribution, or allow others to do so, for U.S.

Government purposes."

\section{RECEIVED \\ DEC 081995 \\ OS.TI}

\author{
Prepared by \\ SOLID STATE DIVISION \\ OAK RIDGE NATIONAL LABORATORY \\ Managed by \\ LOCKHEED MARTIN ENERGY SYSTEMS \\ under \\ Contract No. DE-AC05-84OR21400 \\ with the \\ U.S. DEPARTMENT OF ENERGY \\ Oak Ridge, Tennessee
}

June 1995

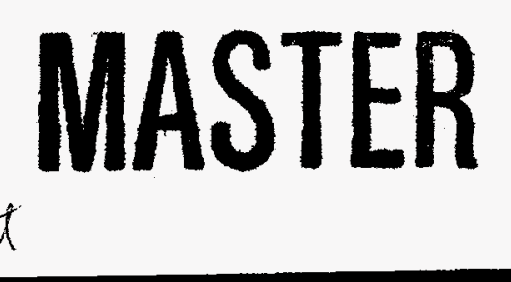




\section{DISCLAIMER}

Portions of this document may be illegible in electronic image products. Images are produced from the best available original document. 


\title{
Determination of Interface Structure and Bonding by Z-Contrast STEM
}

\author{
S. J. Pennycook, N. D. Browning, M. M. McGibbon, A. J. McGibbon, \\ M. F. Chisholm, and D. E. Jesson
}

Oak Ridge National Laboratory, Solid State Division

Oak Ridge, Tennessee 37831-6030

\begin{abstract}
$\underline{\text { ABSTRACT }}$
The scanning transmission electron microscope (STEM) provides a route for the determination of interface structure and bonding directly from experimental data. Through an annular detector, $Z$-contrast images reveal atomic column locations without prior knowledge. The incoherent nature of such images allows a direct structure inversion through a maximum entropy analysis. The Z-contrast image also facilitates atomic-resolution spectroscopy by allowing the probe to be positioned with atomic precision. With this combination of atomic-resolution imaging and spectroscopy, structural units for [001] tilt grain boundaries in $\mathrm{SrTiO}_{3}$ were identified. All units revealed the presence of half-filled columns, an efficient way to overcome the problem of like-ion repulsion in ionic materials. With the $1.3 \AA$ probe of the 300-kV STEM, an unexpected core structure has been found for Lomer dislocations at a CdTe/ GaAs [001] interface, while $60^{\circ}$ dislocations were directly identified to be of glide type.
\end{abstract}

\section{INTRODUCTION}

High-resolution scanning transmission electron microscopes can today form probes as small as $1.3 \AA$, sufficient, using the $Z$-contrast mode, to resolve and distinguish the sublattice in compound semiconductors. The schematic in Fig. 1 shows the essential components of the STEM. Opposite from its conventional counterpart, the objective lens, the critical element in the optical system, is placed before the specimen and used to form a small probe. This probe may be of atomic dimensions, as seen in Fig 2, where parameters are chosen appropriate for the VG Microscopes HB501UX (100 kV) and HB603 $(300 \mathrm{kV})$ as installed at Oak Ridge National Laboratory. The simulated images show the expected appearance of silicon viewed along the [110] axis. The image is formed by scanning the probe and using the 


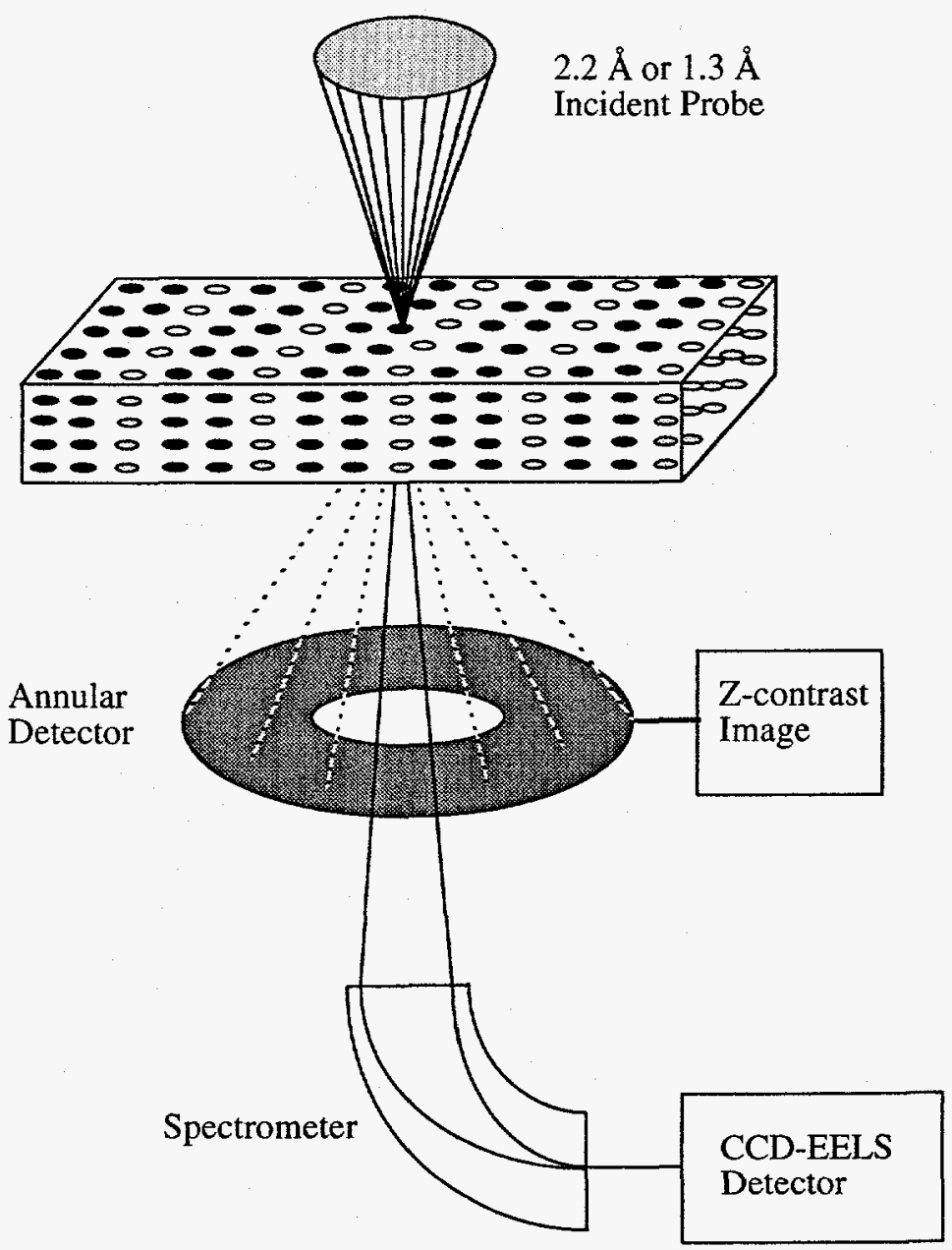

Fig. 1. Schematic showing the optical arrangement for atomic imaging and analysis with the scanning transmission electron microscope.

annular detector to collect scattered electrons. These images have a very different character from the phase contrast images of conventional high-resolution microscopes. The annular detector collects electrons scattered over a wide range of angles, and therefore gives an incoherent image [1-6]. Atomic columns are seen with a brightness dependent on their scattering power, roughly proportional to $Z^{2}$, where $Z$ is atomic number. The key advantage of incoherent imaging is that the microscope can just be focussed for maximum image contrast; then the bright spots show the atomic columns directly without relying on image simulations. Interface structures may be simply read from the image, as shown by Fig. 3 for a $\mathrm{CoSi}_{2} / \mathrm{Si}(111)$ interface [7]. An incoherent image removes the need for preconceived model structures, and often reveals unexpected interface complexity.

This strategy is completely opposite to that of conventional high-resolution electron microscopy, where the goal is the precise measurement of phases. Here we try to destroy any phase correlations, except those depending on probe position, by averaging over the annular detector, which results in an image whose intensity is directly correlated with atomic column positions. In thicker crystals, the angular 
a
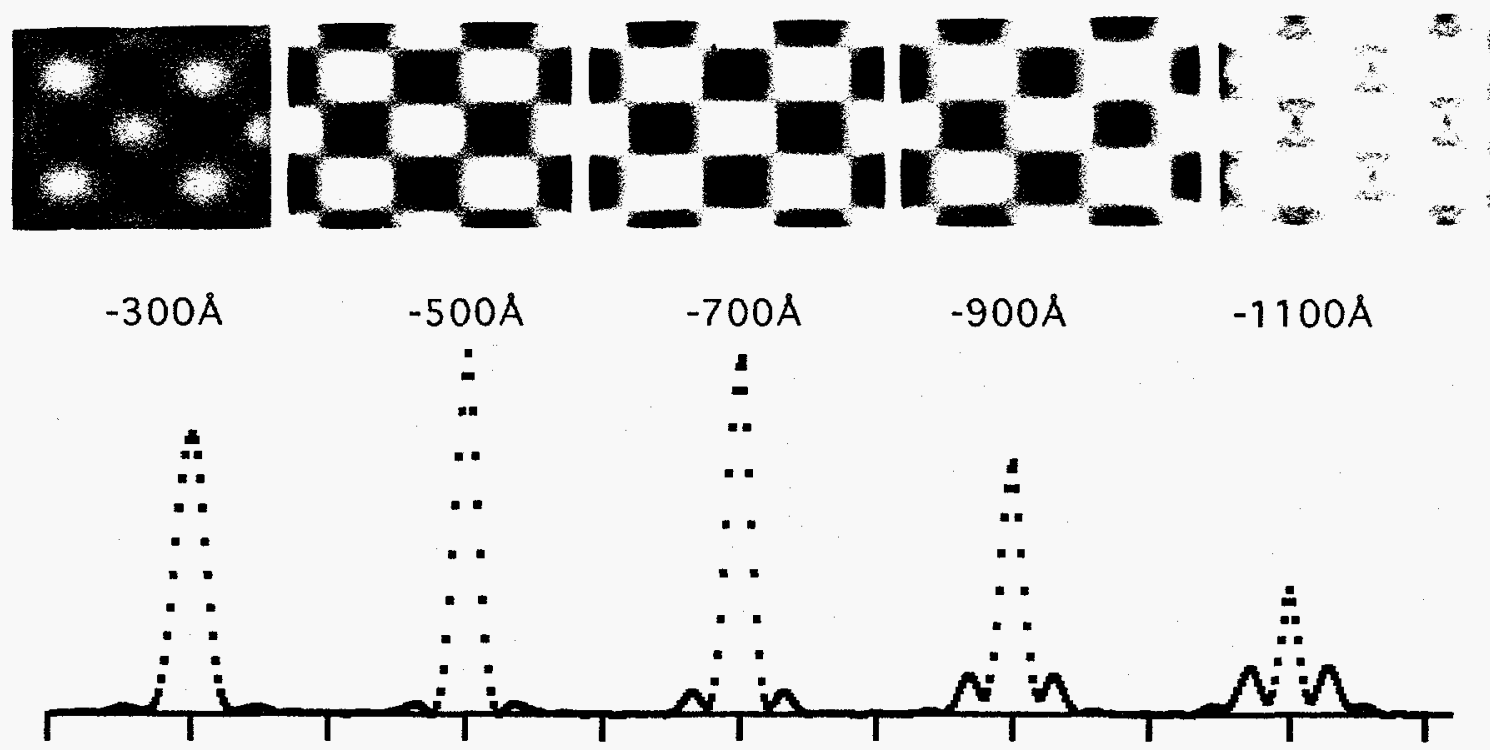

b
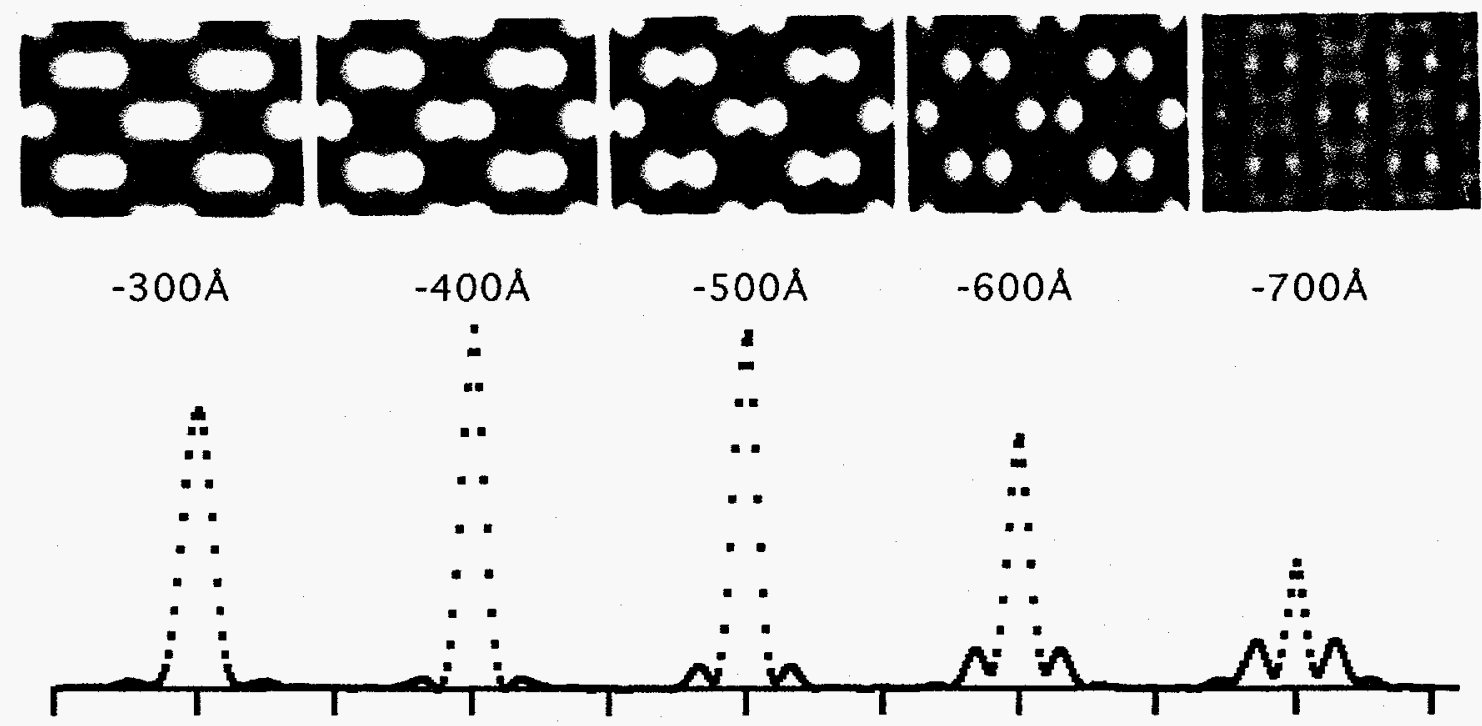

$$
-400 A
$$$$
-500 \AA
$$$$
-600 \AA
$$$$
-700 \AA
$$

Fig. 2. Simulated through focal series for $\mathrm{Si}\langle 110\rangle$ with corresponding probe intensity profiles for (a) the $100-\mathrm{kV}$ STEM (optimum probe size $2.2 \AA$ ), (b) the $300-\mathrm{kV}$ STEM (optimum probe size $1.3 \AA$ ), predicting resolution of the column pairs or "dumbbells". Each profile is shown from $-5 \AA$ to $5 \AA$.

spread of the probe itself is an important contributor to the incoherent characteristics. The dynamical diffraction effects inherent in a conventional phase contrast image are averaged by this angular integration, and incoherent characteristics are retained. Physically, one can simply imagine that part of the probe channels along each column, and the remaining electrons do not contribute to the image contrast. The channelled fraction depends on the probe intensity at the top of the column, so as the probe scans we effectively have column-by-column illumination, the basis for column-by-column imaging and column-by-column analysis. 


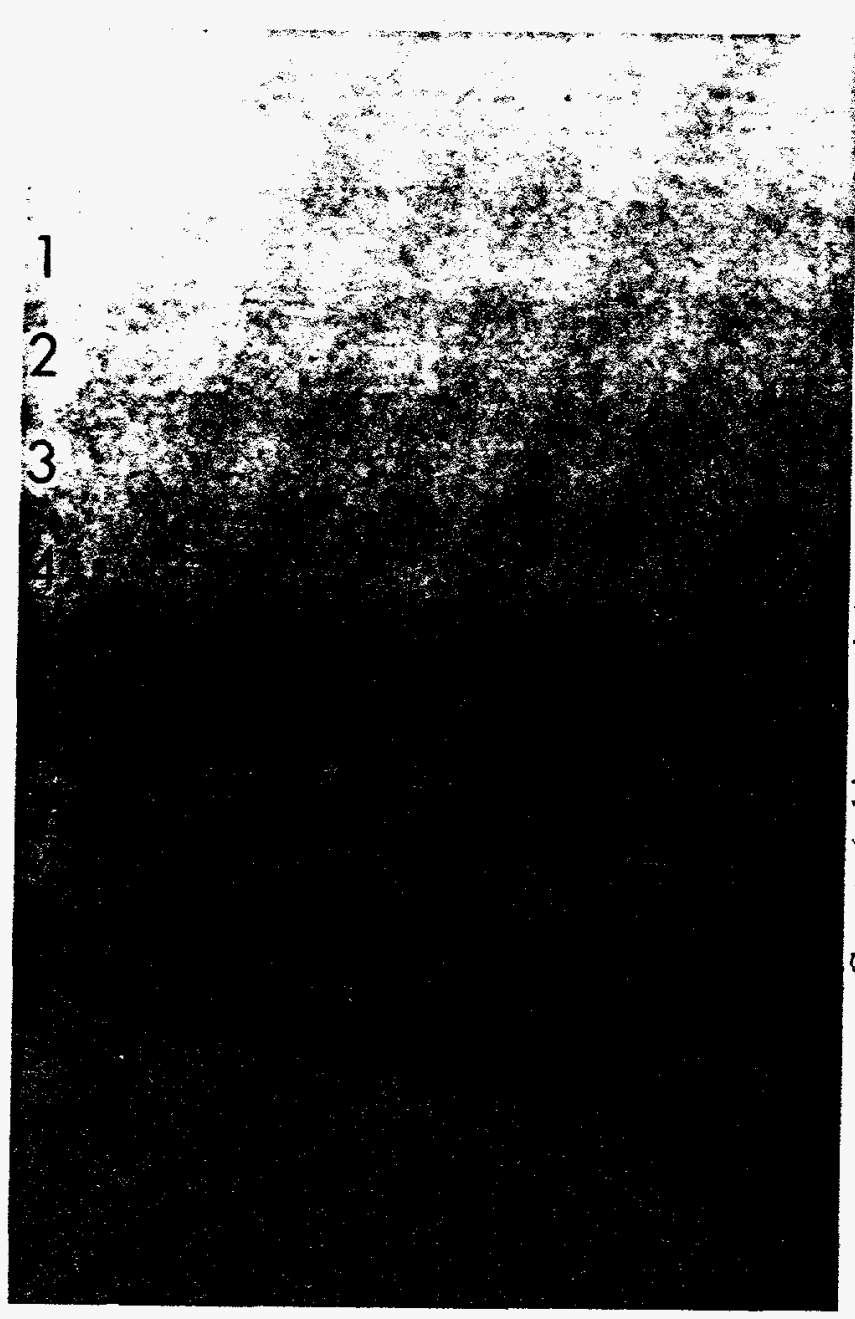

Fig. 3. Z-contrast image of an epitaxial $\mathrm{CoSi}_{2} / \mathrm{Si}(111)$ interface, taken with the $100-\mathrm{kV}$ microscope, allowing direct determination of the interface structure [7].

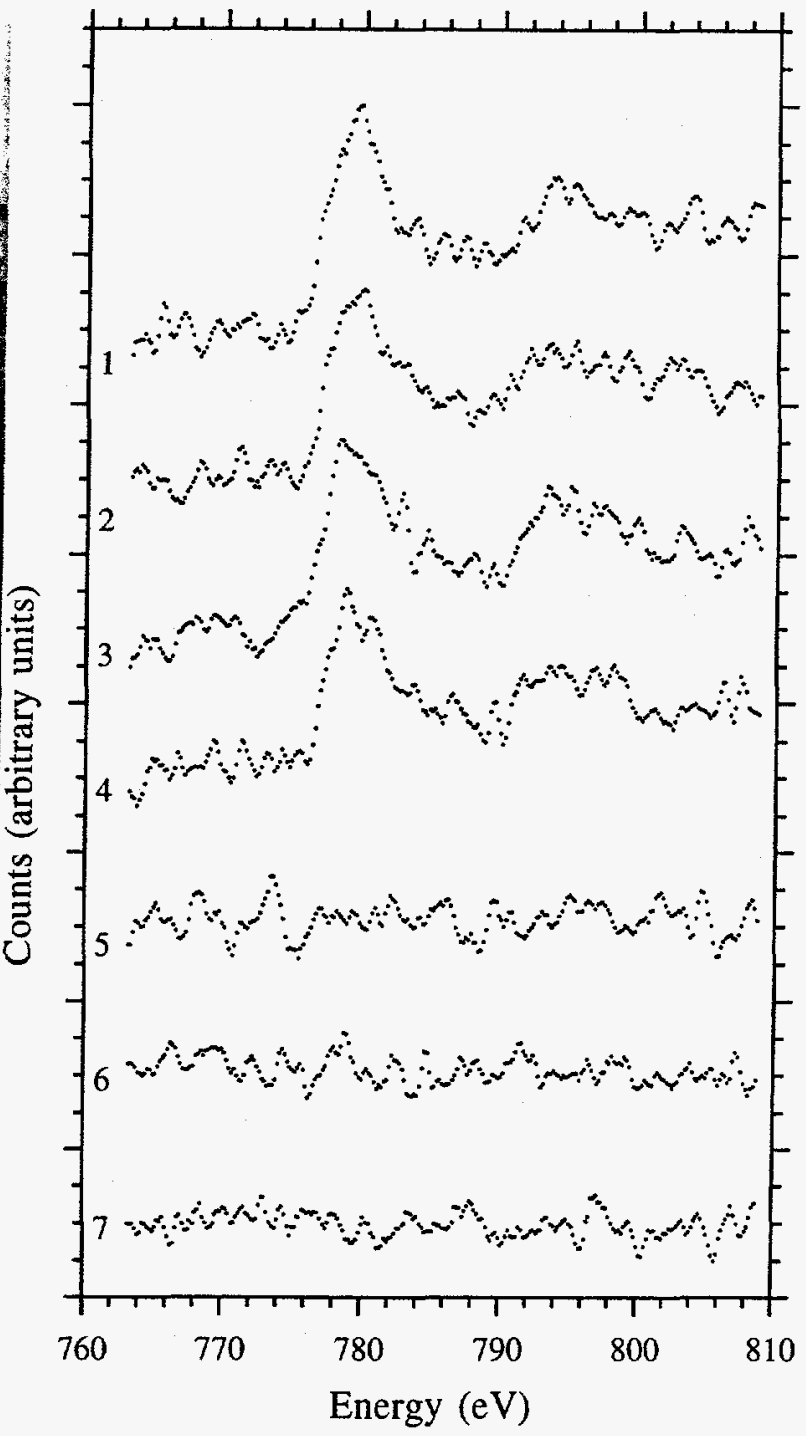

Fig. 4. Co L spectra recorded plane by plane across the interface shown in Fig. 3, demonstrating atomicresolution analysis with a resolution of $2.7 \AA[10]$.

It is apparent from Fig. 1 that provided the inelastically scattered electrons are collected over a large angular range, incoherent imaging conditions will also apply $[8,9]$. However, due to the delocalisation of inelastic energy transfers, to achieve an atomic-resolution analysis it is necessary to use quite high energy losses. Then the inelastic cross sections are much smaller than those for elastic scattering, too small to allow imaging directly. In this case, since both signals may be collected simultaneously, provided incoherent conditions apply to both, the Z-contrast image can be used as a reference image to position the probe for atomic-resolution spectroscopy [10]. Figure 4 shows spectra acquired in this way across the silicide interface of Fig 3. Clearly, the Co-L edge disappears on moving from the last plane of the silicide to the first plane of the silicon, demonstrating that atomic resolution chemical analysis is possible by such means. To compensate for the low current in 
the atomic-sized probe and to avoid beam damage due to long exposures, we use a highly efficient $C C D$ detection system designed by McMullan [11]. Note that the use of a large (30 mrad) spectrometer collection aperture degrades the width of the fine structure to about $5 \mathrm{eV}$.

While our sensitivities are only of the few \% level, because we only illuminate a small number of atoms, we expect to have single atom detectability in thin crystals. Therefore, for dopants or impurities that segregate to some recognisable feature, such as a dislocation core, we now have the possibility of detecting segregation below the level at which precipitation occurs. It should even be feasible to map segregation atomic column by atomic column to match with elasticity or electronic structure calculations and so to achieve finally an experimental probe of the electronic effects of individual dislocations.

\section{DIRECT DETERMINATION OF INTERFACE STRUCTURES}

The incoherent nature of the $Z$-contrast image, and the fact that we expect a discrete lattice of localized scatterers, makes a maximum entropy analysis particularly attractive. As we do not have the phase problem of conventional structure determination methods, this strategy therefore provides a direct structure inversion. Using the method of Gull and Skilling [12], we retrieve the most likely object consistent with the image, with no prior knowledge except for the probe profile. An approximate probe profile can of course be estimated from the image itself. Figure 5 shows this procedure applied to a $\mathrm{SrTiO}_{3}$ image obtained with the 100-kV STEM. The method is robust towards specimen tilts, microscope misalignments and the exact probe defocus, and provides column coordinates with an accuracy of around $0.2 \AA$ [13]. Intensity information is also preserved in the retrieved object, as seen in Fig. $5 \mathrm{~b}$ where the $\mathrm{Sr}$ column locations are seen as brighter dots than the Ti columns. This object can then be convoluted with a small Gaussian to give a reconstructed image as shown in Fig. 5c.

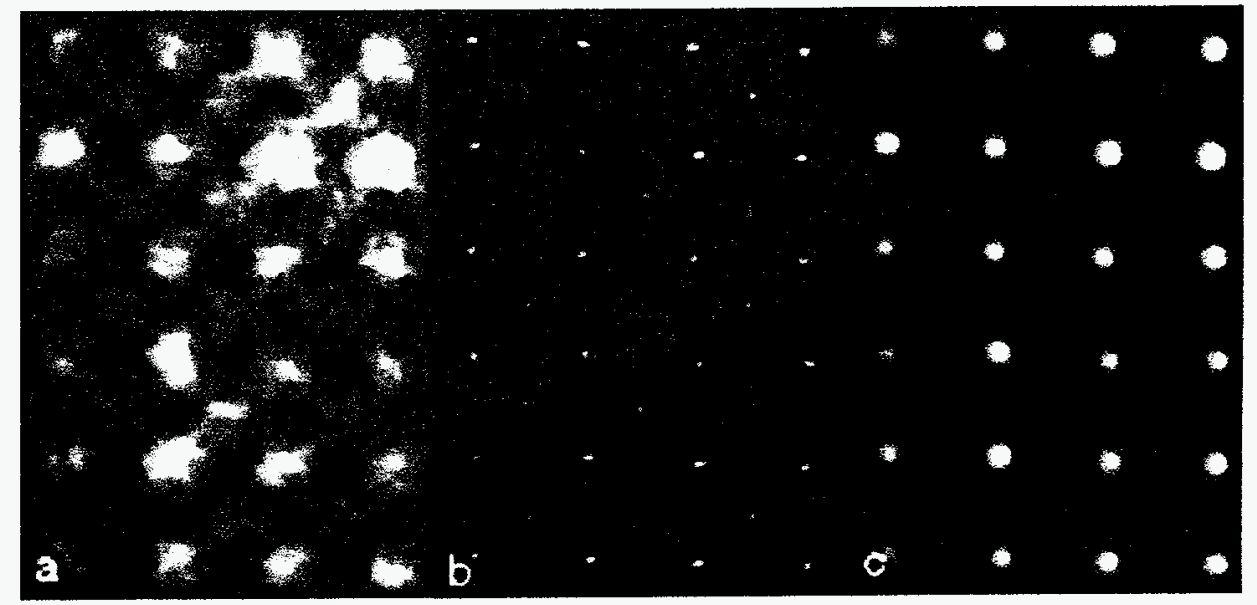

Fig. 5. (a) Z-contrast image of $\mathrm{SrTiO}_{3}$ viewed along the [001] direction; the brightest spots correspond to the Sr columns, separation $3.9 \AA$, the less bright spots are Ti-O columns. (b) Maximum entropy retrieval of the most likely object given only the probe profile. (c) convolution of the retrieved object with a small Gaussian to form a noise-reduced maximum entropy image which retains the position and intensity information of the original image. 
For the investigation of grain boundaries, it now becomes possible to determine structure models directly from the experimental data. As an example, Fig. 6 shows the maximum entropy image of a $25^{\circ}$ symmetric [001] tilt grain boundary in a $\mathrm{SrTiO}_{3}$ bicrystal [14]. The cation coordinates are retrieved from the maximum entropy analysis, while the oxygen coordination can be studied by electron energy loss spectroscopy (EELS). Spectra recorded from the grain boundary plane showed no major departures from octahedral $\mathrm{Ti}-\mathrm{O}$ coordination, though evidence of bond distortion was found. The $\mathrm{O}$ atoms were therefore placed in octahedral positions and the structure refined using bond valence sums to give the final structure shown in Fig $6 \mathrm{~b}$.

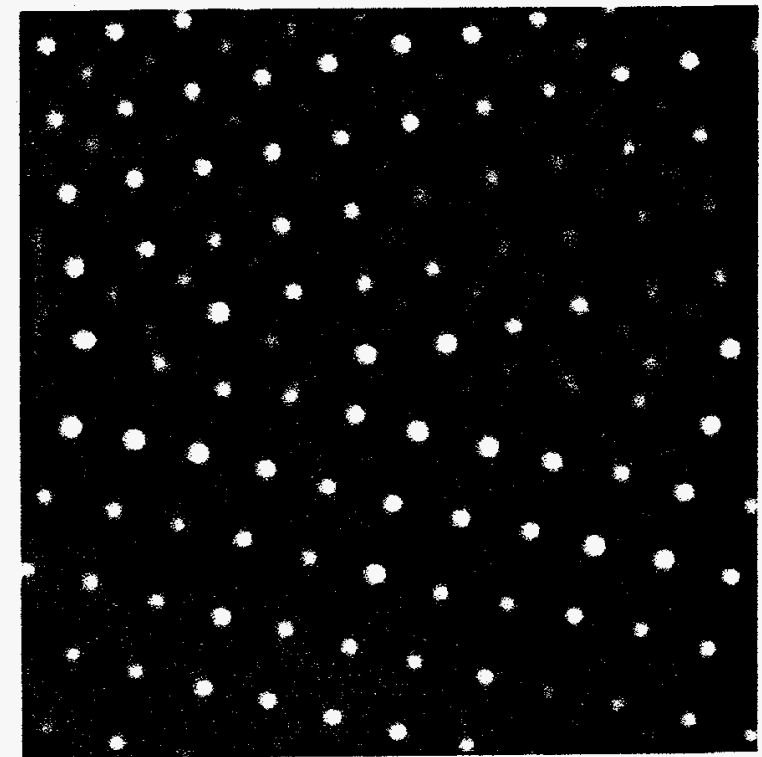

a

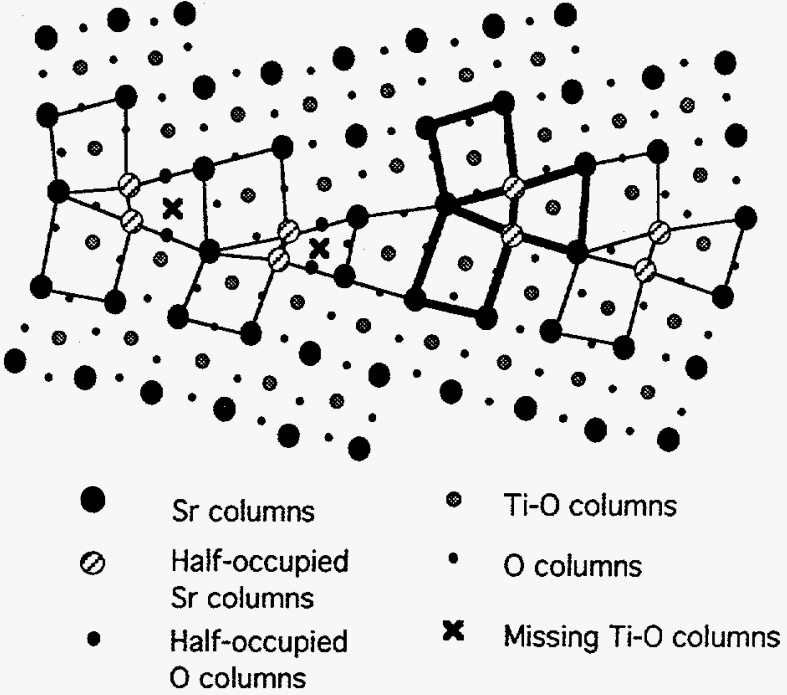

b

Fig. 6. (a) Maximum entropy image of a $25^{\circ}$ symmetric [001] tilt grain boundary in a $\mathrm{SrTiO}_{3}$ bicrystal. (b) Deduced atomic structure. Note the microscopic asymmetry of the boundary structure.

Several unexpected features were found at this boundary, most significantly, the presence of half-filled $\mathrm{Sr}$ columns shown hatched. The incoherent nature of the $Z$-contrast image definitively indicates two scattering centers approximately $2 \AA$ apart, both of which would appear to be $\mathrm{Sr}$ from their positions in their respective halves of the bicrystal, but clearly they cannot coexist at that spacing if fully occupied. The half- or zigzag columns represent the simplest model consistent with the experimental data, and these columns do show reduced intensity as expected, although quantification of intensities at the boundary plane is not sufficiently accurate to determine a precise occupancy.

Also somewhat surprising is the observation of the triangular structural units forming this $\{920\}$ boundary, since they represent primitive dislocation cores and therefore introduce a microscopic asymmetry into the boundary structure, which can be considered as microfacetting. Furthermore, two variants of the basic structural unit were found, with and without a central Ti-O column. The reason for this is not clear, but relaxations of the neighboring cells correlated with the presence or absence of the Ti-O column. A relatively large expansion was measured normal 
to the boundary plane, $0.6 \pm 0.2 \AA$. Together with the half columns this represents the grain boundary excess volume. One can also speculate on the likely atomic sites for impurity atoms; clearly, the $\mathrm{Sr}$ ions in the half-filled columns have a larger atomic volume than in the bulk, and so represent likely sites for substitution by large cations, while the relaxations around the Ti-O columns implies they could accomodate dopants of different valence.

Applying the same method to $36^{\circ}$ and $65^{\circ}$ symmetric tilt boundaries, further structural units were identified, again all characterized by the presence of half-filled columns. These additional structural units were sufficient to construct models for any symmetric [001] tilt grain boundary [15], using the method of Sutton and Vitek [16]. However, a $45^{\circ}$ symmetric bicrystal showed that the boundary had decomposed into a set of small asymmetric facets suggesting that long period symmetric grain boundaries may be unstable. Again, however, two structural units could be identified, and were found to coexist in all asymmetric grain boundaries [17].

Such an approach, combining $Z$-contrast imaging with EELS analysis, is equally applicable to other systems. With high-temperature superconductors, for example, the hole concentration can be measured directly by EELS, without having to cool the specimen, with a spatial resolution below the superconducting coherence length [18]. This allows the local superconducting properties to be correlated with the actual atomic structure; preliminary results have demonstrated strong hole depletion at an asymmetric boundary, whereas none was observed at a symmetric boundary. Such effects may well be explained by the structural unit approach.

\section{STRUCTURE IMAGING WITH THE 300-kV STEM}

The 1.3- $\AA$ probe of the $300-\mathrm{kV}$ STEM means that all semiconductor materials can now be studied by the same strategy. It is possible to examine Si bicrystals to determine grain boundary structures, while EELS could be used to investigate dopant segregation, for example As. It is finally conceivable, for example, to achieve a true atomic level understanding of the fast diffusion paths in polycrystalline $\mathrm{Si}$. At present we have no spectrometer on the $300-\mathrm{kV}$ instrument; such studies would need data from both machines. There is no reason however that the 300-kV STEM could not be equipped with a suitable high efficiency spectrometer.

The main motivation for the 300-kV STEM has been the possibility of directly resolving and distinguishing the sublattice in compound semiconductors. Figure 7 compares maximum entropy images from $\mathrm{Si}$ and from $\mathrm{GaAs}$ in which the sublattice polarity is clearly visible. GaAs represents the most severe test, since $\mathrm{Ga}$ and As are only two atomic numbers apart, although they are expected to show $\sim 10 \%$ intensity difference, sufficient to allow the polarity to be distinguished. At present, due to shot noise, image statistics limit the accuracy of intensity measurements and occasionally individual dumbbells are seen with inverted polarity. Thus a polarity determination requires more than one individual dumbbell.

Nevertheless, it has proved possible to determine core structures for misfit dislocations in compound semiconductor heterostructures. The lattice polarity is 


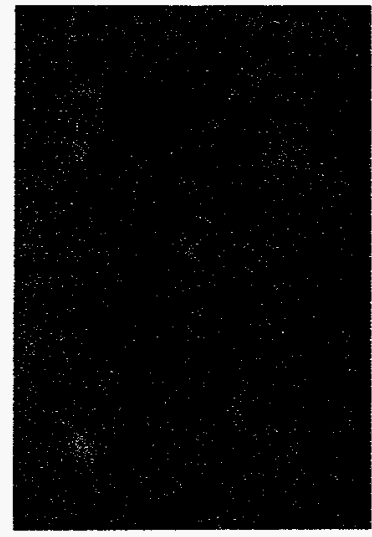

a

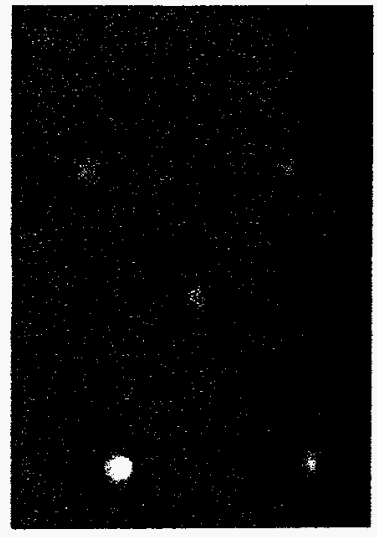

b

Fig. 7. 300-kV Z-contrast images of (a) Si (b) GaAs after maximum entropy restoration revealing lattice polarity.

deduced several dumbbells away from the dislocation core, then compositions in the core region are assigned accordingly. In this way, it has proved possible to distinguish glide and shuffle dislocations directly, and to determine the terminating species in the core [19]. Figure 8 shows core structures of $60^{\circ}$ dislocations at a $\mathrm{CdTe} / \mathrm{GaAs}(001)$ interface, in the two perpendicular $\langle 110\rangle$ directions. Both dislocations are seen to be of glide type, one terminating at the $\mathrm{Cd}$ column, the other at the Te column. Figure 9 shows images of perfect edge (Lomer) dislocations from the same specimen, also in the two perpendicular $\langle 110\rangle$ directions, one showing a core structure similar to that proposed originally by Hornstra [20], the other showing an unexpected core structure comprising a four-fold ring surrounded by distorted six-fold rings. Only the Hornstra core is located at the interface plane; all others are located a few monolayers into the CdTe film. It can be speculated that the more polar CdTe does not like the 5- or 7-membered rings of the Hornstra core. When the Hornstra core occurs, the like-ion bonds are $\mathrm{Ga}-\mathrm{Ga}$ bonds, although the preference for one core structure over another can only become clear after theoretical investigations of the energetics of these structures. Certainly images such as these do have the power to reveal unanticipated effects; they do therefore represent firm starting points for theoretical studies of this nature.

\section{SUMMARY}

The advances of the last few years have now given us the tools with which to probe electronic materials at the true atomic scale, atomic column by atomic column, not only intrinsic structures such as dislocation cores, but also the nature and distribution of impurity segregation. We can anticipate an increasing correlation of such data with theoretical modelling of structure and transport characteristics; indeed, the structure models we can deduce experimentally represent a firm foundation for further theoretical work, which should lead at last to a real, atomic level understanding of the electronic and optical properties of semiconductor materials. 


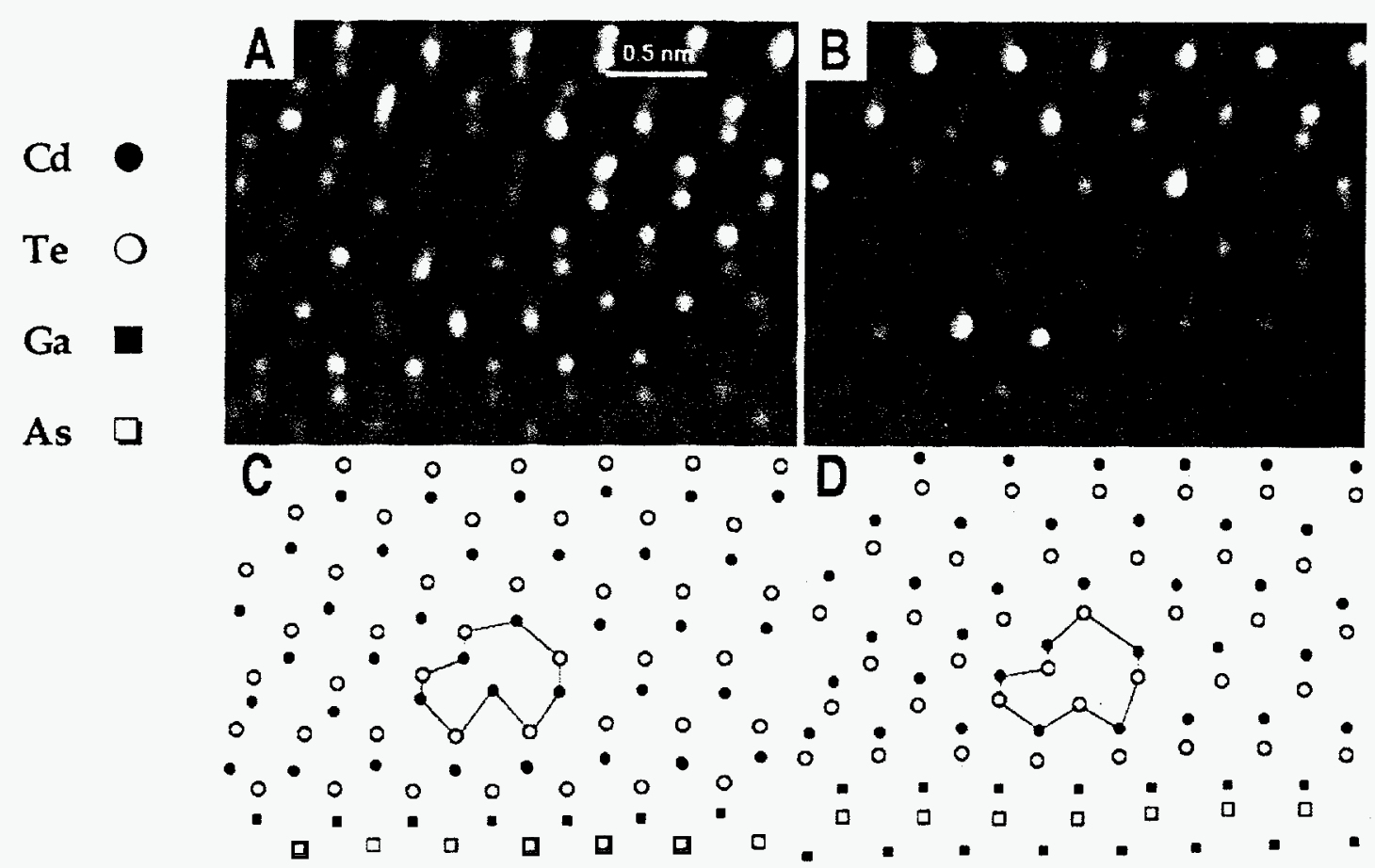

Fig. 8. Maximum entropy images of $60^{\circ}$ dislocations at the $\mathrm{CdTe}(001) / \mathrm{GaAs}(001)$ interface viewed in (a) [110] and (b) [110] orientations, with schematic representations given in (c) and (d) respectively. It can be seen that both dislocations are of the glide set, and are located a few monolayers above the interface.

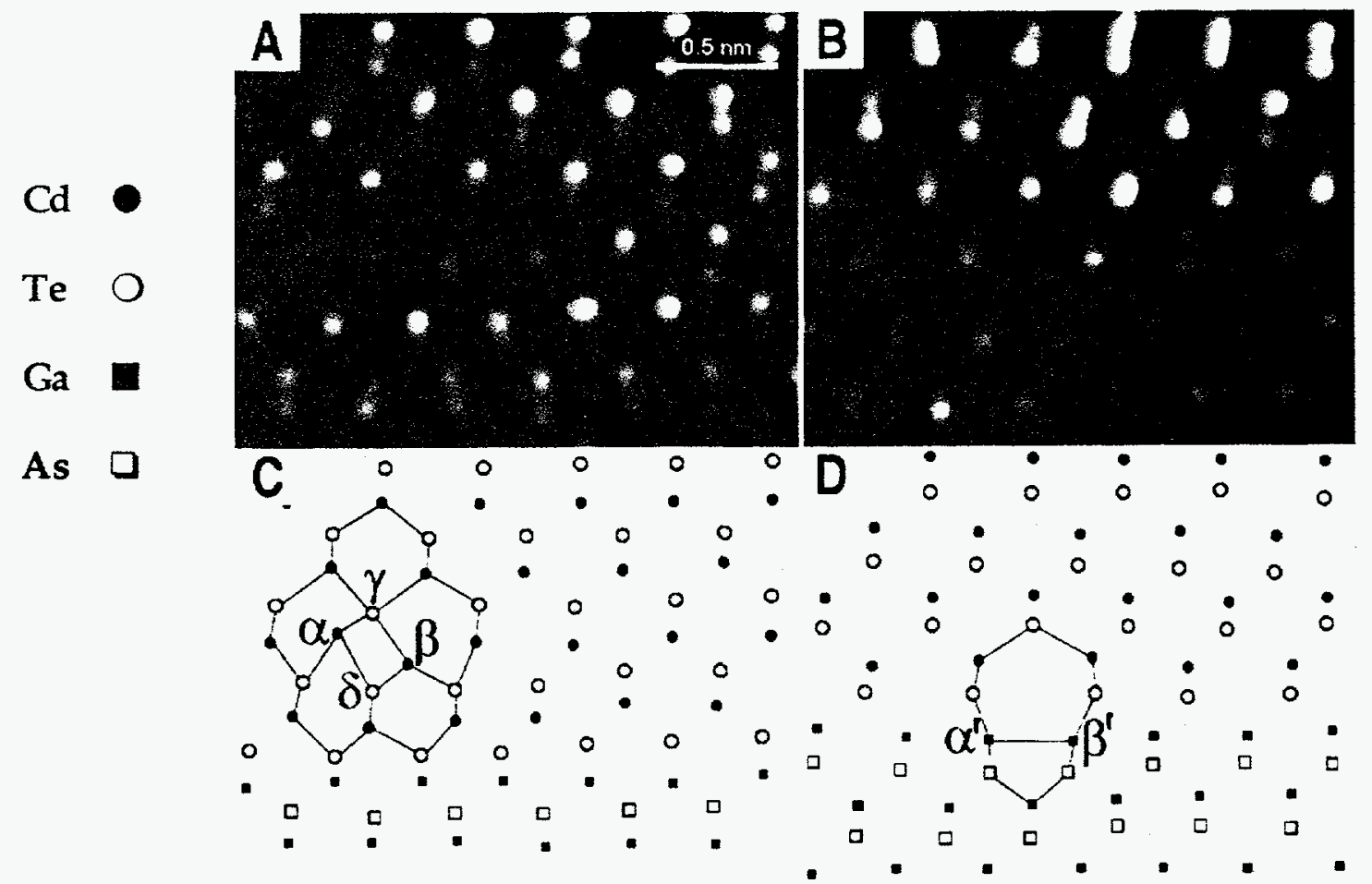

Fig. 9. Lomer dislocations at the interface of CdTe(001)/GaAs(001) viewed in the (a) [110] and (b) [110] orientations, with schematic representations given in (c) and (d) respectively. The dislocation in (a) lies a few monolayers above the interface and possesses an unexpected core structure whilst that in (b) lies at the interface and has the Hornstra structure. 


\section{ACKNOWLEDGEMENT}

The authors are grateful to R. Jebasinski, S. Mantl, V. P. Dravid, V. Ravikumar and J. E. Angelo for provision of samples, and to T. C. Estes, J. T. Luck, and S. L. Carney for technical assistance. This research was sponsored by the Division of Materials Sciences, U.S. Department of Energy, under contract DE-AC05-84OR21400 with Lockheed Martin Energy Systems and in part by appointments to the Oak Ridge National Laboratory Postdoctoral Research Program administered by the Oak Ridge Institute for Science and Education.

\section{REFERENCES}

1. Pennycook, S. J. and Jesson, D. E.: Phys. Rev. Lett., 1990, 64, 938.

2. Pennycook, S. J. and Jesson, D. E.: Ultramicroscopy, 1991, 37, 14.

3. Pennycook, S. J. and Jesson, D. E.: Acta Metall. Mater., 1992, 40, S149.

4. Jesson, D. J., and Pennycook, S. J.: Proc. R. Soc. Lond. A, 1993, 441, 261.

5. Jesson, D. J., and Pennycook, S. J.: Proc. R. Soc. Lond. A, 1995, 449, 273.

6. Pennycook, S. J., Browning, N. D., Jesson, D. E., Chisholm, M. F., and McGibbon, A. J.: Appl. Phys. A, 1993, 57, 385.

7. Chisholm, M. F., Pennycook, S. J., Jebasinski, R., and Mantl, S.: Appl. Phys. Lett., 1994, 64, 2409.

8. Ritchie, R. H. and Howie, A.: Phil. Mag. A, 1988, 58, 753.

9. Pennycook, S. J., Jesson, D. E., and Browning, N. D.: Nucl. Instrum. and Methods Phys. Res. B (in press).

10. Browning, N. D., Chisholm, M. F., and Pennycook, S. J.: Nature, 1993, 366, 143.

11. McMullan, D., Rodenburg, J. M., Murooka, Y., and McGibbon, A. J.: Institute of Physics Conference Series, 1990, 98, 55.

12. Gull, S. F. and Skilling, J.,: IEE Proceedings, 1984, 131, 646.

13. McGibbon, A. J., Pennycook, S. J., and Jesson, D. E.: J. Microscopy (in press).

14. McGibbon, M. M., Browning, N. D., Chisholm, M. F., McGibbon, A. J., Pennycook, S. J., Ravikumar, V., and Dravid, V. P.: Science, 1994, 266, 102.

15. Browning, N. D., Pennycook, S. J., Chisholm, M. F., McGibbon, M. M., and McGibbon, A. J.: Interface Science, 1995, 2, 397. 
16. Sutton, A. P. and Vitek, V.: Phil. Trans. R. Soc. Lond. A, 1983, 309, 1.

17. McGibbon, M. M., Browning, N. D., McGibbon, A. J., and Pennycook, S. J.: Phil. Mag. A (submitted).

18. Browning, N. D., Chisholm, M. F., Pennycook, S. J., Norton, D. P., and Lowndes, D. H.: Physica C, 1993, 212, 185.

19. McGibbon, A. J., Pennycook, S. J., and Angelo, J. E.: Science (in press).

20. Hornstra, J.: J. Phys. Chem Solids, 1958, 5, 129.

\section{DISCLAIMER}

This report was prepared as an account of work sponsored by an agency of the United States Government. Neither the United States Government nor any agency thereof, nor any of their employees, makes any warranty, express or implied, or assumes any legal liability or responsibility for the accuracy, completeness, or usefulness of any information, apparatus, product, or process disclosed, or represents that its use would not infringe privately owned rights. Reference herein to any specific commercial product, process, or service by trade name, trademark, manufacturer, or otherwise does not necessarily constitute or imply its endorsement, recommendation, or favoring by the United States Government or any agency thereof. The views and opinions of authors expressed herein do not necessarily state or reflect those of the United States Government or any agency thereof. 